ARTICLE

Received 7 Jun 2013 | Accepted 15 Jan 2014 | Published 12 Feb $2014 \quad$ DOl: 10.1038/ncomms4260

\title{
Polariton-generated intensity squeezing in semiconductor micropillars
}

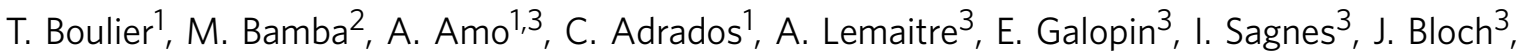 \\ C. Ciuti $^{4}$, E. Giacobino ${ }^{1} \&$ A. Bramati ${ }^{1}$
}

The generation of squeezed and entangled light fields is a crucial ingredient for the implementation of quantum information protocols. In this context, semiconductor materials offer a strong potential for the implementation of on-chip devices operating at the quantum level. Here we demonstrate a novel source of continuous variable squeezed light in pillarshaped semiconductor microcavities in the strong coupling regime. Degenerate polariton four-wave mixing is obtained by exciting the pillar at normal incidence. We observe a bistable behaviour and we demonstrate the generation of squeezing near the turning point of the bistability curve. The confined pillar geometry allows for a larger amount of squeezing than planar microcavities due to the discrete energy levels protected from excess noise. By analysing the noise of the emitted light, we obtain a measured intensity squeezing of $20.3 \%$, inferred to be $35.8 \%$ after corrections.

\footnotetext{
${ }^{1}$ Laboratoire Kastler Brossel, Université Pierre et Marie Curie, Ecole Normale Supérieure and CNRS, UPMC Case 74, 4 place Jussieu, 75252 Paris Cedex 05, France. ${ }^{2}$ Department of Physics, Osaka University, 1-1 Machikaneyama, Toyonaka, Osaka 560-0043, Japan. ${ }^{3}$ Laboratoire Photonique et Nanostructures, CNRS, Route de Nozay, 91460 Marcoussis, France. ${ }^{4}$ Laboratoire Matériaux et Phénomènes Quantique, Université Paris Diderot et CNRS, Bâtiment Condorcet, 10 rue Alice Domon et Léonie Duquet, 75205 Paris Cedex 13, France. Correspondence and requests for materials should be addressed to A.B. (email: bramati@spectro.jussieu.fr).
} 
W hile very efficient sources of non-classical light, essentially based on bulk nonlinear crystals, have been developed in the last 20 years, the quest for integrated quantum sources is a major challenge for the cutting edge research activity in the field of quantum information technologies.

Successful examples in the discrete variable regime are represented by efficient single and entangled photons sources in semiconductor quantum dots ${ }^{1-3}$. Photon antibunching and squeezing were theoretically predicted and experimentally demonstrated in pioneering resonance fluorescence experiments $^{4-7}$. In strong contrast, despite several theoretical proposals ${ }^{8,9}$, the continuous variables regime has seen so far only very few experimental achievements ${ }^{10-12}$ of squeezed states of light in semiconductor materials.

Here, for the first time in this type of systems, we achieve a high degree of intensity squeezing exploiting an effective Kerr effect for microcavity polaritons. Let us note that, due to the giant polariton nonlinearities ${ }^{13}$, the power range used here $(5-20 \mathrm{~mW})$ is two orders of magnitudes lower than in previous experiments ${ }^{10}$. These results open the way to the implementation of ultra-low threshold quantum devices integrated in a semiconductor platform.

Exciton-polaritons are half-light half-matter bosonic particles arising from the strong coupling between excitons and photons in semiconductor microcavities ${ }^{14}$. They exhibit strong nonlinearities due to the Coulomb interactions of their excitonic part, responsible for the rich variety of quantum effects recently unveiled $^{15}$, ranging from Bose-Einstein condensation ${ }^{16}$ and superfluidity ${ }^{17,18}$ to more complex phenomena such as dark and bright solitons ${ }^{19,20}$, magnetic monopole-like half-solitons ${ }^{21}$, half-vortices ${ }^{22}$ and vortex lattices ${ }^{23}$.

The great potential of polaritons for non-classical fields generation was recognized very soon after the experimental demonstration of the coherent nonlinear optical response of microcavity polaritons allowing for the observation of parametric amplification and oscillation in such systems ${ }^{24-26}$. Especially interesting is the case of degenerate polariton four-wave mixing in which the strong nonlinearities originating in the polaritonpolariton interactions allow describing the polariton system as an effective Kerr medium whose refractive index depends on the light intensity ${ }^{12,27,28}$. Kerr media are well known to be very efficient sources of quadrature-squeezed light. Indeed, quadrature squeezing of about $4 \%$ was observed in a planar semiconductor microcavity, by using degenerate polariton four-wave mixing in 2004 (ref. 11). Despite several attempts with specifically designed planar microcavity samples, significant improvements of this first result were prevented by the excess noise coming from the intrinsic multimode nature of the emission in a planar microcavity, which is characterized by a continuum of polariton modes and is favourable to the coupling with lattice phonons. Very recently, it has been predicted ${ }^{29}$ that a large amount of squeezing could be achieved by quantizing the in-plane polariton field modes via a geometric confinement (strong localization limit ${ }^{29}$ ). This tight confinement separates the modes energetically, severely reducing any nonlinear multimode coupling responsible for increased excess noise and reduced quantum effects. In this paper, we apply this method in a controlled way making use of semiconductor micropillars ${ }^{30,31}$ and we demonstrate up to $20.3 \%$ of directly measured squeezing in the amplitude quadrature of the emitted light.

\section{Results}

Experimental configuration. In our set-up, the pillars are fabricated by etching a $\lambda$ GaAs microcavity containing two
GaAs-InGaAs quantum wells, resulting in a polariton Rabi splitting of about $4 \mathrm{meV}$. The cavity finesse is of the order of 700, which gives a $0.1 \mathrm{meV}$ polariton linewidth. Figure 1 shows the energy separation of the polariton modes due to the finite size of the pillar. As shown in Fig. 2a, the cavity mirrors are asymmetric with the bottom Bragg mirror twice thicker than the top one (30 pairs of $\mathrm{Al}_{0.1} \mathrm{Ga}_{0.9} \mathrm{As}-\mathrm{Al}_{0.95} \mathrm{Ga}_{0.05}$ As versus 15 pairs for the top mirror), optimizing the cavity for experiments in reflection configuration. The cavity is wedged in one direction, providing a large choice of cavity-exciton detuning by selecting pillars placed in different positions on the chip. We can choose the shape and size of the micropillars in order to engineer the spatial distribution of the confined modes and their energy separation. Figure $2 b$ shows the large square-shaped pillars (width of $20 \mu \mathrm{m}$ ) and small square or round-shaped pillars (width from $6 \mu \mathrm{m}$ to $2 \mu \mathrm{m}$ ) available on the studied sample.

The pillars are cooled down to $5 \mathrm{~K}$ in a cryostat mounted in reflection configuration and excited with a CW single-mode Ti:Sa laser tuned to be quasi-resonant with the ground-state level of the lower polariton branch. The laser is frequency locked to a Fabry-Perot cavity and its intensity is stabilized by means of an electro-optical modulator. The laser was verified to be at the shot noise for frequencies above $1 \mathrm{MHz}$. The spatial mode is filtered by a five meters long single-mode optical fibre shielded against thermal fluctuations. The laser beam is focused by a microscope objective treated with a near-infrared optimized coating and excites the sample at normal incidence. Upstream of the microscope objective, a motorized $\frac{\lambda}{2}$ plate coupled with a polarizing beam splitter (PBS) allows us to finely control the pump power on the sample and a $\frac{\lambda}{4}$ plate placed between the beam splitter and the objective ensures that the light coming on the sample is circularly polarized. With the polarized beam splitter, the $\frac{\lambda}{4}$ plate forms an optical circulator since the light reemitted from the sample was measured to be $95 \%$ circularly polarized, as expected from spin conservation. The light reflected from the cavity is thus collected and sent to the detectors.

The shape of the laser spot on the sample is Gaussian with a waist of $5.5 \mu \mathrm{m}$, well matched to the round pillars with a diameter of $6 \mu \mathrm{m}$. In the studies presented here, we hence use a round micropillar of $6 \mu \mathrm{m}$, which ensures an optimal mode matching between its polaritonic ground state and the focalized excitation

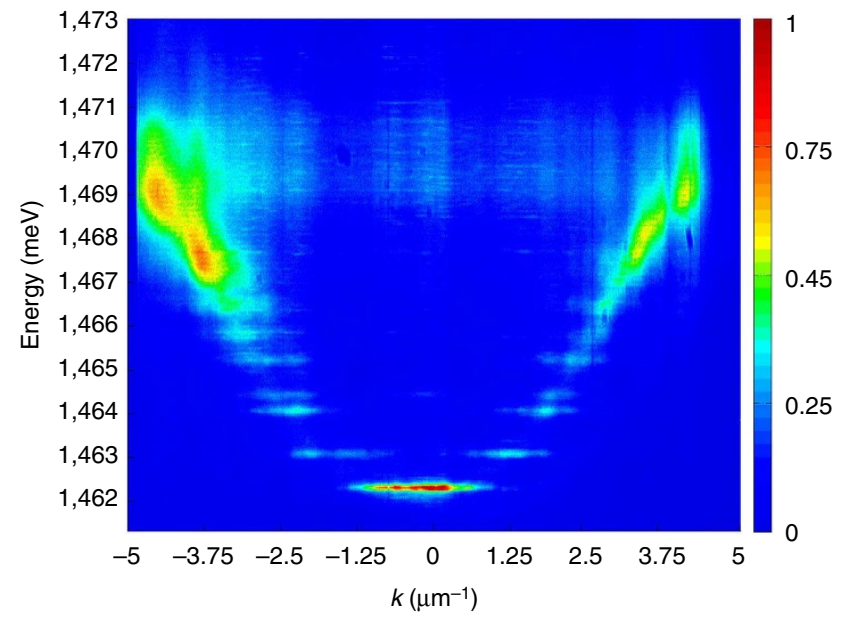

Figure 1 | Energy modes separation. Dispersion curve for the $6 \mu \mathrm{m}$ circular pillar used in the experiment ( $-5 \mathrm{meV}$ cavity detuning), obtained with a non-resonant pumping at 1,550 meV. The lower polariton branch with several spatial modes and the weakly excited upper branch are visible. The modes are energetically separated and squeezing is protected against multimode nonlinear coupling. 
a

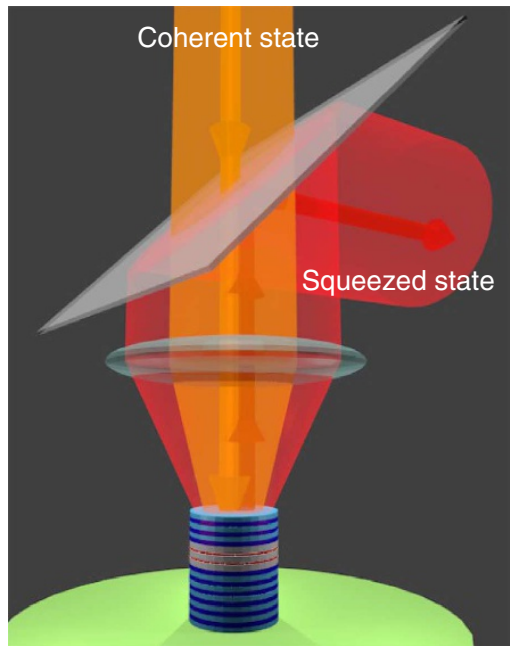

b

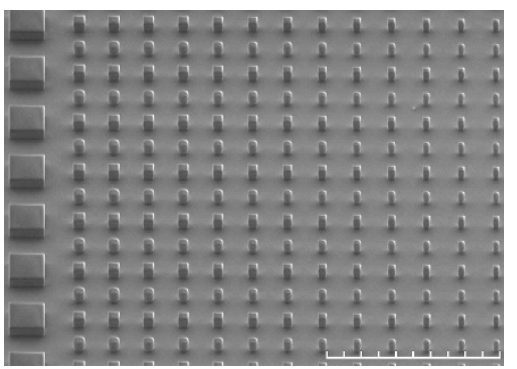

C

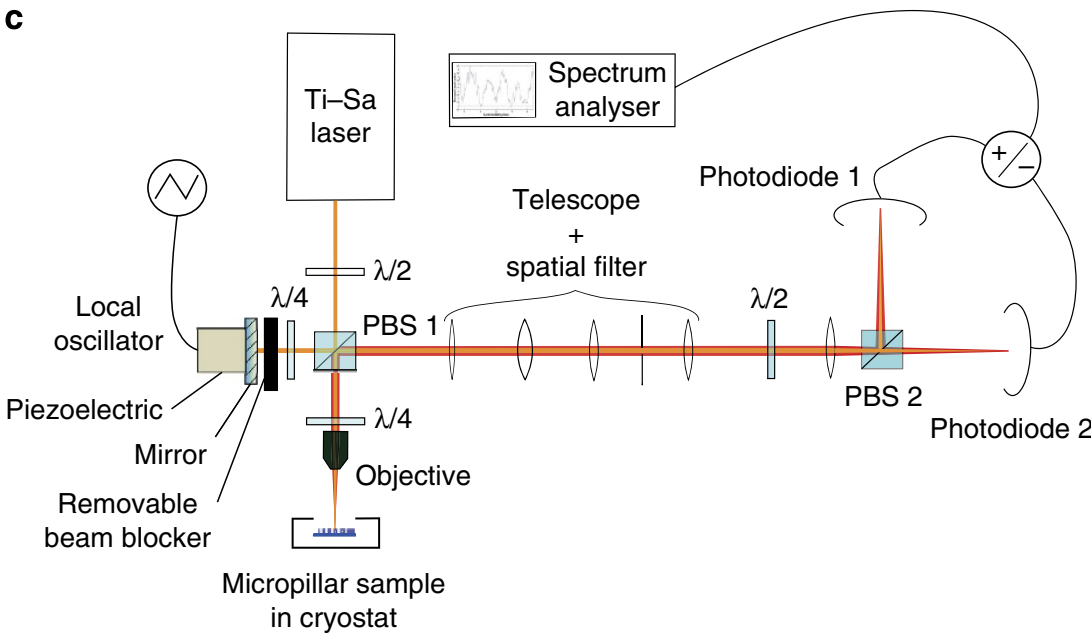

Figure 2 | Sample and set-up. (a) Principle of squeezed light production with a pillar-shaped microcavity. The microcavity is made of a pair of quantum wells (white-red-white layers) sandwiched by two Bragg mirrors (alternation of light blue and dark blue layers); the green layer is the substrate. The orange beam represents the pump laser arriving on the sample at normal incidence, slightly blueshifted with respect to the energy of the lower polariton branch. The red beam represents the squeezed light emitted by the squeezed polariton field present in the microcavity. (b) Image of the sample obtained with scanning electron microscopy. The different shapes and sizes of the pillars are visible. The scale bar corresponds to $100 \mu \mathrm{m}$. (c) Scheme of the experimental set-up for the noise detection. The higher squeezing values were measured with the balanced detection set-up, realized by blocking the local oscillator with the beam blocker. The choice of adding $(+)$ or subtracting $(-)$ the photodiodes signals determines the measurement: intensity noise $(+)$ or shot noise $(-)$. To measure the noise quadratures of the field one unblocks the local oscillator and set the mixer to $(-)$; in this case, the shot noise is measured by blocking the light reflected from the microcavity. PBS: polarizing beam splitter; $\frac{\lambda}{2}, \frac{\lambda}{4}$ : half-wave, quarter-wave plate.

laser. The re-emitted light is collected by the same microscope objective and sent through a telescope and a spatial filter, allowing us to select only the light coming out of the pillar or some specific region if needed. As shown in Fig. 2c, the sample emission is collected in a set-up allowing performing both balanced and homodyne detections.

For the balanced detection, the collected light is equally split into two parts by a $\frac{\lambda}{2}$ plate associated to a a polarizing beam splitter (PBS 2) and each beam is collected by a photodiode. The high frequency (HF) outputs of the two identical photodiodes (81\% quantum efficiency, with home-made low-noise electronics) are added or subtracted with a passive circuit, amplified by a lownoise, HF amplifier and sent to a spectrum analyser giving us the spectral noise power (thereafter simply called 'noise'). The choice of addition or subtraction allows us to quickly switch between the measurement of the intensity noise (addition mode) or the shot noise (difference mode). The common mode rejection of the balanced diodes was $45 \mathrm{~dB}$ at $7 \mathrm{MHz}$.

For the homodyne detection, a portion of the pump laser is mixed on PBS 1 with the light emitted by the micropillar with an orthogonal polarization, and acts as local oscillator. In this case, the HF output of the photodiodes are subtracted and the measured quantity gives the noise in the quadrature of the reflected field that is in phase with the local oscillator. The relative phase between the local oscillator and the reflected field is controlled with a piezoelectric-mounted mirror, allowing measuring all quadratures. The shot noise is measured by blocking the light reflected from the microcavity.

For the homodyne set-up, it is important to note that the spatial mode of the sample emission can be strongly distorted by the nonlinear interactions and it is no longer Gaussian-shaped at high polariton densities. For this reason, it was found very 
difficult to achieve the mode matching with the local oscillator necessary to have efficient homodyne detection, except in the special case of very low detuning of the pump laser with respect to the ground-state level of the lower polariton branch (since in this case we have a low density of polariton even above threshold). There, proper homodyne detection was achieved (mode matching of $90 \%$ ). However, such a low detuning is not optimal and thus the squeezing measured with the homodyne detection is lower than at the optimum detuning.

Intensity squeezing measurements at the optimum pumppolariton detuning were performed with the balanced set-up. Figure 3a,b shows the intensity noise measured with a balanced detection: a scan at fixed pump power in Fig. 3a and a scan of the noise versus pump power in Fig. $3 \mathrm{~b}$ for a polariton-laser detuning $\Delta=0.19 \mathrm{meV}$ that we have identified as optimal for squeezing. In Figs 3 and 4, the spectrum analyser was set to zero frequency span and centred at $7 \mathrm{MHz}$ where the signal-to-noise ratio of the detection is maximum, with a sweep time ranging from $1 \mathrm{~s}$ to $20 \mathrm{~s}$ depending on the type of measurement. The resolution bandwidth (spectral bandwidth of the measurement) was $300 \mathrm{kHz}$ with a $30 \mathrm{~Hz}$ video bandwidth (temporal integration over a $30 \mathrm{~Hz}$ bandwidth). For the noise dependence on the pump power (Fig. 3b), the motorized plate controlling the excitation power on the pillar is scanned at the same time as the spectrum analyser. We then compare the measured noise to the shot noise. The protocol involves recording the shot noise in difference mode, immediately switching to addition mode to record the intensity noise and then switching back to shot noise measurement (difference mode again) in order to check its stability. The dark noise was recorded after each measurement by recording the electronic noise when the laser beam is blocked. All the presented noise measurements are obtained by normalizing the noise traces by the shot noise after subtraction of the dark noise from both the noise and the shot noise.

A slight energy detuning between the pump laser and the ground state of the lower polariton branch gives rise to optical bistability $^{12}$ (see Fig. 3c), characteristic in Kerr nonlinear media. According to theoretical predictions, the best squeezing rate is expected in the vicinity of the turning points of the bistability, as in other nonlinear systems ${ }^{32}$. We therefore chose to sweep the pump power in the vicinity of the bistable region (noise profile measurement, Fig. 3b) to identify the best working points and we performed noise measurements with a fixed pump power corresponding to the maximum of intensity squeezing (steadystate measurement, Fig. 3a).

Squeezing was achieved in a reproducible way in repeated experiments on several different neighbouring pillars. According to previous calculations ${ }^{33}$, the best squeezing is expected for a slightly negative cavity-exciton detuning $(0>\delta>-1 \mathrm{meV})$ in planar microcavities. Here the spatial confinement of pillars tends to enhance the polariton-polariton nonlinear interactions with respect to planar microcavities due to the increase in polariton density. We hence chose to work at larger negative cavity-exciton detuning $(\delta \approx-5 \mathrm{meV})$ to have a larger photonic fraction, while preserving strong enough nonlinearities from the excitonic component.

Experimental and theoretical results. The strongest noise reduction at fixed pump power was obtained with the laser energy blue-detuned by about $\Delta=0.19 \mathrm{meV}$ from the polariton lower energy level on a $6 \mu \mathrm{m}$ pillar with a cavity-exciton detuning at $\delta \approx-5 \mathrm{meV}$. The measured intensity squeezing is $20.3 \pm 1.5 \%$, as shown in Fig. 3a. After correction for detection efficiency (81\%) and transmission losses $(T=70 \%)$, we infer a squeezing in the outgoing field of $35.8 \pm 2.6 \%$. a
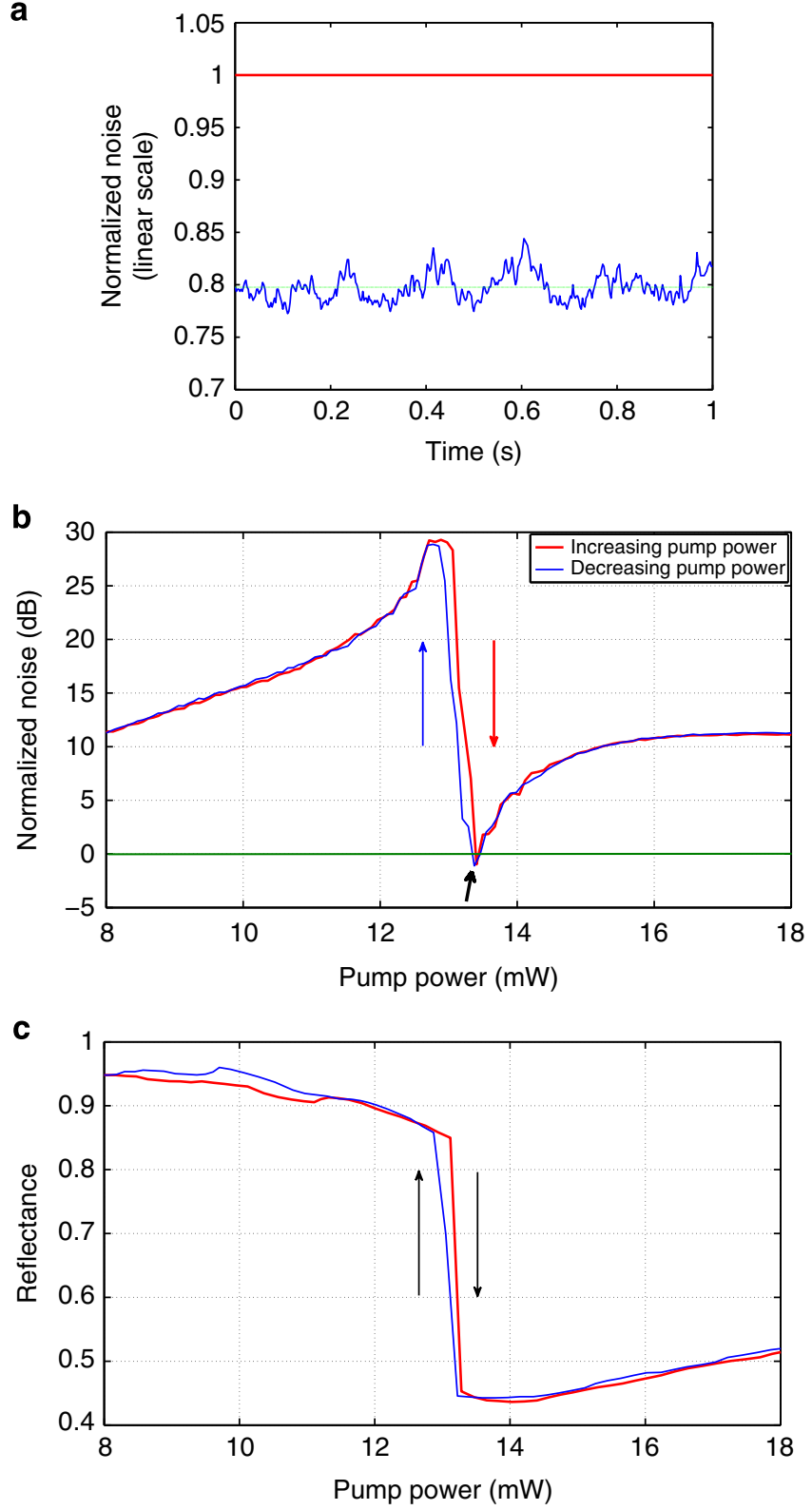

Figure 3 | Intensity squeezing and bistability. (a) Squeezed intensity noise at fixed pump power near the lower turning point (linear scale), on a $6 \mu \mathrm{m}$ pillar at cavity-exciton detuning of $-5 \mathrm{meV}$ and a polariton-laser detuning of $0.19 \mathrm{meV}$. The noise data were normalized with a measurement of the shot noise and a measurement of the dark noise, with $S(t)=$ (noise(t) $-<\operatorname{dark}(t)>) /(<\operatorname{shotnoise}(t)>-<\operatorname{dark}(t)>),<\ldots>$ being the time average. The measured noise reduction is $20.3 \pm 1.5 \%(-1 \mathrm{~dB})$ below the shot noise limit before corrections, $35.8 \pm 2.6 \%$ after. The dashed green line is the average noise and the red line is the shot noise. (b) Intensity noise (spectral noise power, semi-logarithmic scale) as a function of the pump power for the same pillar under the same conditions as in $\mathbf{a}$. The noise increases smoothly towards a peak just before the bistability threshold, then suddenly drops to form a gap where intensity squeezing is observed, with about the same value as in $\mathbf{a}$. Here also the normalized spectral noise is obtained with three measurements (noise, shot noise and dark noise). In red is the curve obtained by increasing the pump power, in blue is the curve obtained by decreasing the pump power and the green line represents the shot noise (normalized to one). The thick black arrow points to where a was recorded. (c) Bistability curve obtained by plotting the reflectance versus the pump power, for the same pillar under the same conditions as in $\mathbf{a}, \mathbf{b}$. The colour code is the same as in $\mathbf{b}$. Above the thresholds the transmission is increased, hence a sharp reduction on the reflectance. 


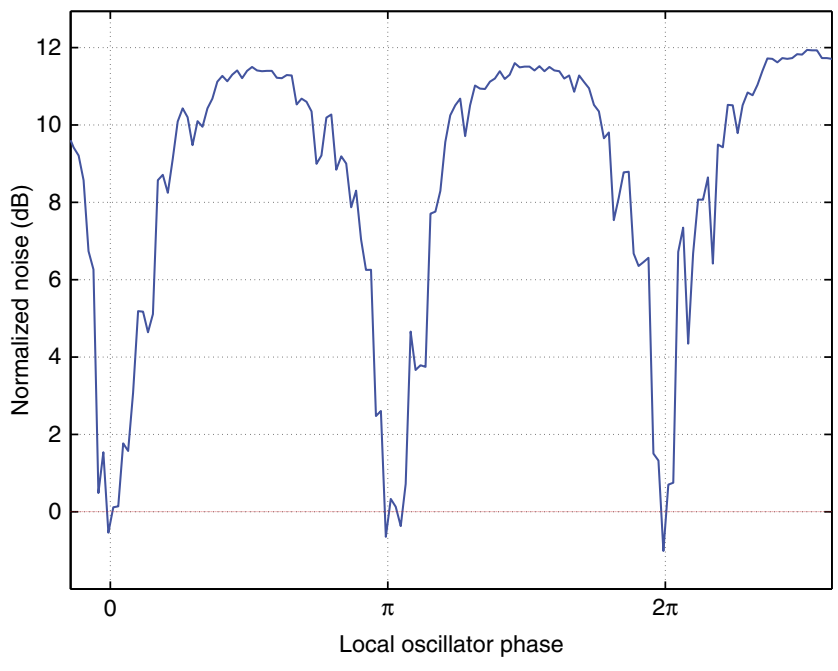

Figure 4 | Homodyne detection. Experimental measurement of squeezing with homodyne detection for a pump-polariton detuning of $0.10 \mathrm{meV}$ on the same pillar as in Fig. 3. The minima were verified to be in the amplitude quadrature. The squeezing obtained from the average between the values at $0, \pi$ and $2 \pi$ is $16 \%$ ( $-0.75 \mathrm{~dB}$ ) before corrections and $25 \%$ after corrections. The reduced value of the squeezing here is mainly due to the non-optimal laser-polariton detuning.

This result was established with a laser intensity lock reducing the low frequency $(0-100 \mathrm{kHz})$ intensity variations to $0.2 \%$. The use of the spatial filter was found not to be crucial, as the laser spot was well matched to the size of the six pillars. On the other hand, excessive spatial filtering led to a reduction of the squeezing, as expected from increasing losses. A coupled excitonphoton model with pump, losses and Kerr nonlinearity (described in Methods) predicts a squeezing value under these conditions of about $40 \%$, in agreement with the experimental value.

Effect of the pump-polariton detuning. The values of pump power at which the optimal working points occur as well as the specific shape of the bistability curve depend on $\Delta$. This parameter is also expected to influence the intensity noise profile and the squeezing value. The noise dependence on the pump power for the same pillar and parameter values as mentioned above is shown in Fig. 3b. Outside of the bistable region there is an excess noise due to thermal exciton reservoir populated by the pump laser. In the vicinity of the bistability thresholds, this noise undergoes amplification or deamplification due to the nonlinear effects. The intensity noise strongly increases below the bistability thresholds then decreases, as observed in other nonlinear systems ${ }^{15,32,33}$.

At higher values of $\Delta$, the pump power required to reach the bistability threshold increases and the noise profiles are drastically altered. Indeed a bistable behaviour appears above some critical value of $\Delta$. For rather large $\Delta$ only the lower turning point (reached by decreasing the pump power) is able to produce intensity squeezing. If $\Delta$ is lower than the critical value, strong nonlinear modifications of the noise and reflectance can be observed despite the lack of bistable behaviour and the noise curves are the same when increasing or decreasing the pump power. These effects were studied in our experiment by repeating the noise measurements at different polariton-laser detunings and are discussed in the Supplementary Note 1. Supplementary Figs 1 and 2 show, respectively, the reflectance and the noise plots obtained for $\Delta=0.10 \mathrm{meV}$ while Supplementary Figs 3 and 4 show, respectively, the reflectance and the noise plots obtained for $\Delta=0.21 \mathrm{meV}$ and Supplementary Figs 5 and 6 show the reflectance and the noise plots obtained for $\Delta=0.30 \mathrm{meV}$. The optimal detuning for squeezing that we present here corresponds to the onset of the bistability behaviour where the hysteresis cycle is very narrow (Fig. 3b).

Homodyne detection. To confirm these findings, we have measured the noise along all the field quadratures with the homodyne detection and checked that squeezing occurs only in the amplitude quadrature at the bistability turning points. As previously mentioned, for low $\Delta$ the output of the pillar is nearly Gaussian. In such conditions, we were able to achieve good mode matching between the local oscillator and the spatial mode emitted by the micropillar (about 90\%) and implement an efficient homodyne detection. The path difference between the two beams, set to change linearly with time, determines the measured quadrature. The result is presented in Fig. 4 for a laser-polariton detuning of $\Delta=0.10 \mathrm{meV}$. Squeezing is observed at the minima of the curve showing the characteristic arches. Let us note that the antisqueezed quadrature (phase quadrature) exhibits a large excess noise of the order of $12 \mathrm{~dB}$ due to thermal noise input. The fluctuations and irregularities of the noise arches are due to the fact that the interferometric homodyne set-up is more sensitive to mechanical instabilities. A comparison with the intensity measurements set-up allows checking that the squeezed quadrature indeed corresponds to the amplitude quadrature. The observed squeezing with this set-up was of about $16 \%$ (25\% inferred after corrections).

\section{Discussion}

In conclusion, we have reported an experimental demonstration of strong enhancement of squeezed light generation with confined polaritons in semiconductor micropillars. The best intensity squeezing was inferred to be $35.8 \pm 2.6 \%$ below the shot noise limit and was confirmed by measurements with a homodyne detection set-up. Several original optimizations allowed to reach these results. In particular, the special design of the pillars ensures a near-perfect mode matching between the pump and the polariton field; the discretization of the polariton energy modes allows for a strong reduction of the excess noise coming from phonon coupling. The reduced reflectivity of the upper Bragg mirror relative to that of the bottom mirror enhances the emission of light towards the top of the pillar, optimizing the light collection. Finally the relatively low-quality factor of the cavity increases the radiative to non-radiative losses ratio which, together with the choice of a working point with a high photonic fraction, results in a larger squeezing in the emitted light. These results demonstrate the improvement of intensity-squeezed light and light-matter fields brought by reduced dimensionality in semiconductor microstructures and open the way to the realization of ultra-low threshold integrated sources for the quantum information-processing technology.

\section{Methods}

Theoretical model. Owing to the lateral confinement of the micropillar, the polariton modes are well identified and we can selectively pump a polariton mode by tuning the spatial and spectral profile of the pumping light. For the theoretical analysis, we simply consider a single-cavity mode with frequency $\omega_{\mathrm{c}}$ and an exciton state with frequency $\omega_{\mathrm{x}}$. The Hamiltonian of the coupled system (polariton) in the microcavity is:

$$
\hat{H}_{0}=\hbar \omega_{\mathrm{c}} \hat{a}^{\dagger} \hat{a}+\hbar \omega_{\mathrm{x}} \hat{b}^{\dagger} \hat{b}+\frac{\hbar \Omega_{\mathrm{R}}}{2}\left(\hat{a}^{\dagger} \hat{b}+\hat{b}^{\dagger} \hat{a}\right)+\frac{\hbar g}{2} \hat{b}^{\dagger} \hat{b}^{\dagger} \hat{b} \hat{b}
$$

Here, $\hat{a}$ and $\hat{b}$ are the annihilation operators of a photon and an exciton, respectively, and $\Omega_{R}$ is the coupling strength (vacuum Rabi splitting) between the cavity photon and the exciton. The last term represents a third-order nonlinearity 
for the excitons with strength $g$. The polaritons are subject to an effective Kerr effect through it. In addition, considering the photonic and excitonic reservoirs, the quantum Langevin equations for the cavity photons and the excitons are, respectively, derived as follows:

$$
\begin{gathered}
i \frac{\partial \hat{a}}{\partial t}=\left(\omega_{\mathrm{c}}-\frac{i \kappa}{2}\right) \hat{a}+\left(\frac{\Omega_{\mathrm{R}}}{2}\right) \hat{b}-i \sqrt{\kappa}\left(\hat{a}_{\mathrm{in}}+F_{\mathrm{p}} e^{-i \omega_{\mathrm{p}} t}\right) \\
i \frac{\partial \hat{b}}{\partial t}=\left(\omega_{\mathrm{x}}-\frac{i \gamma}{2}\right) \hat{b}+\left(\frac{\Omega_{\mathrm{R}}}{2}\right) \hat{a}+g \hat{b}^{\dagger} \hat{b} \hat{b}-i \sqrt{\gamma} \hat{b}_{\mathrm{in}}
\end{gathered}
$$

Here, $\kappa$ and $\gamma$ are the dissipation rates of the cavity photon and exciton, respectively. $\hat{a}_{\text {in }}$ and $\hat{b}_{\text {in }}$ are the input operators from photonic and excitonic reservoirs, respectively, and we assume they have a zero average $\left\langle\hat{a}_{\text {in }}\right\rangle=\left\langle\hat{b}_{\text {in }}\right\rangle=0$ (incoherent input). Instead, we explicitly consider an applied coherent pumping with amplitude $F_{\mathrm{p}}$ and frequency $\omega_{\mathrm{p}}$. Then, the input-output relation for the photon operators is expressed as:

$$
\hat{a}_{\text {out }}=\hat{a}_{\text {in }}+F_{\mathrm{p}} e^{-i \omega_{\mathrm{p}} t}+\sqrt{\kappa} \hat{a}
$$

From the above Langevin equations, we first calculate the mean fields of the cavity photon and exciton induced by the coherent pump. Assuming $\langle\hat{a}\rangle=\alpha e^{-i \omega_{\mathrm{p}} t}$ and $\langle\hat{b}\rangle=\beta e^{-i \omega_{\mathrm{p}} t}$, the steady-state mean fields are as follows:

$$
\begin{aligned}
& i \frac{\partial}{\partial t} \alpha=\left(\omega_{\mathrm{c}}-\omega_{\mathrm{p}}-\frac{i \kappa}{2}\right) \alpha+\left(\frac{\Omega_{\mathrm{R}}}{2}\right) \beta-i \sqrt{\kappa} F_{\mathrm{p}}=0 \\
& i \frac{\partial}{\partial t} \beta=\left(\omega_{\mathrm{x}}-\omega_{\mathrm{p}}-\frac{i \gamma}{2}\right) \beta+\left(\frac{\Omega_{\mathrm{R}}}{2}\right) \alpha+g|\beta|^{2} \beta=0
\end{aligned}
$$

Once the mean fields are determined, the reflectance is calculated via the inputoutput relation as follows:

$$
\begin{aligned}
& \left\langle\hat{a}_{\text {out }}\right\rangle=\alpha_{\text {out }} e^{-i \omega_{\mathrm{p}} t}=\left(F_{\mathrm{p}}+\sqrt{\kappa} \alpha\right) e^{-i \omega_{\mathrm{p}} t} \\
& R=\frac{\left|\left\langle\hat{a}_{\text {out }}\right\rangle\right|^{2}}{\left|\left\langle\hat{a}_{\text {in }}+F_{\mathrm{p}} e^{-i \omega_{\mathrm{p}} t}\right\rangle\right|^{2}}=\frac{\left|F_{\mathrm{p}}+\sqrt{\kappa} \alpha\right|^{2}}{\left|F_{\mathrm{p}}\right|^{2}}
\end{aligned}
$$

The intensity noise is calculated by the fluctuations around the mean field value. Defining the fluctuation operators as $\delta \hat{a}=\hat{a} e^{i \omega_{\mathrm{p}} t}-\alpha$ and $\delta \hat{b}=\hat{b} e^{i \omega_{\mathrm{p}} t}-\beta$, and linearizing the corresponding equations, the quantum Langevin equations are rewritten as follows:

$$
\begin{gathered}
i \frac{\partial}{\partial t} \delta \hat{a}=\left(\omega_{\mathrm{c}}-\omega_{\mathrm{p}}-\frac{i \kappa}{2}\right) \delta \hat{a}+\left(\frac{\Omega_{\mathrm{R}}}{2}\right) \delta \hat{b}-i \sqrt{\kappa} \hat{a}_{\text {in }} \\
i \frac{\partial}{\partial t} \delta \hat{b}=\left(\omega_{\mathrm{x}}+2 g|\beta|^{2}-\omega_{\mathrm{p}}-\frac{i \gamma}{2}\right) \delta \hat{b}+\left(\frac{\Omega_{\mathrm{R}}}{2}\right) \delta \hat{a}+g \beta^{2} \delta \hat{b}^{\dagger}-i \sqrt{\gamma} \hat{b}_{\mathrm{in}}
\end{gathered}
$$

From these equations and the input-output relation, we can infer the fluctuations $\delta \hat{a}_{\text {out }}=\hat{a}_{\text {out }} e^{i \omega_{\mathrm{p}} t}-\alpha_{\text {out }}$ of the output photons. The variance of the output is expressed as follows:

$$
\begin{aligned}
(\Delta N)^{2} & =\left\langle\hat{a}_{\text {out }}^{\dagger} \hat{a}_{\text {out }}^{\dagger} \hat{a}_{\text {out }} \hat{a}_{\text {out }}\right\rangle-\left\langle\hat{a}_{\text {out }}^{\dagger} \hat{a}_{\text {out }}\right\rangle^{2} \\
& =2\left|\alpha_{\text {out }}\right|^{2}\left\langle\delta \hat{a}_{\text {out }}^{\dagger} \delta \hat{a}_{\text {out }}\right\rangle+2 \operatorname{Re}\left[\left(\alpha_{\text {out }}^{*}\right)^{2}\left\langle\delta \hat{a}_{\text {out }} \delta \hat{a}_{\text {out }}\right\rangle\right]
\end{aligned}
$$

Then, the normalized intensity noise is expressed as follows:

$$
\frac{(\Delta N)^{2}}{\left\langle\hat{a}_{\text {out }}^{\dagger} \hat{a}_{\text {out }}\right\rangle}=2\left\langle\delta \hat{a}_{\text {out }}^{\dagger} \delta \hat{a}_{\text {out }}\right\rangle+2 \operatorname{Re}\left[\left(\frac{\alpha_{\text {out }}^{*}}{\left|\alpha_{\text {out }}\right|}\right)^{2}\left\langle\delta \hat{a}_{\text {out }} \delta \hat{a}_{\text {out }}\right\rangle\right]
$$

The correlations of the output are calculated from the ones of input operators. Here, we assume that the correlation of the photonic input as $\left\langle\hat{a}_{\text {in }}^{\dagger}(t) \hat{a}_{\text {in }}\left(t^{\prime}\right)\right\rangle=$ $\left\langle\hat{a}_{\text {in }}(t) \hat{a}_{\text {in }}\left(t^{\prime}\right)\right\rangle=0$ (extracavity photon vacuum) while the excitonic reservoir has an incoherent population, namely $\left\langle\hat{b}_{\text {in }}^{\dagger}(t) \hat{b}_{\text {in }}\left(t^{\prime}\right)\right\rangle=N_{\text {th }} \delta\left(t-t^{\prime}\right)$ and $\left\langle\hat{b}_{\text {in }}(t) \hat{b}_{\text {in }}\left(t^{\prime}\right)\right\rangle=0$. We also assume that there is no correlation between the photonic and excitonic inputs. $N_{\text {th }}$ represents the thermal noise and is chosen to be linear with the pump power $\left(N_{\mathrm{th}}=\sigma \cdot W_{\mathrm{p}}(\mathrm{mW})\right.$, with $\sigma$ the proportionality constant and $W_{\mathrm{p}}$ the pump power).

Parameters. In the calculations, we have used the following values: $\delta=\omega_{\mathrm{c}}-$ $\omega_{\mathrm{x}}=-5 \mathrm{meV}, \Omega_{\mathrm{R}}=4 \mathrm{meV}$ and $\kappa=0.11 \mathrm{meV}$. The other parameters have been estimated by fitting experimental data for several values of $\Delta$, the pump-polariton detuning and are shown in Table 1. From the theoretical results of this simple model, we observe that the excitonic dissipation rate $\gamma$ tends to increase with $\Delta$. This can be explained by an increased population in the excitonic reservoir.

This model is able to reproduce correctly the reflectance traces obtained in the experiment, and the intensity noise for pump intensities above the bistability threshold. Note that the prediction of the intensity noise for low pump intensities does not reproduce well the experimental data. This is probably due to the fact that our model does not take disorder effects into account. For very low densities, the disorder becomes the dominant effect, so we cannot capture the corresponding noise behaviour adequately.
Table 1 | Parameters.

\begin{tabular}{lcc}
$\boldsymbol{\Delta}=\omega_{\mathbf{p}}-\omega_{\text {polariton }}(\mathbf{m e V})$ & $\gamma(\mathbf{m e V})$ & $\boldsymbol{\sigma}\left(\mathbf{m} \mathbf{W}^{-\mathbf{1}}\right)$ \\
\hline 0.14 & 0.43 & 0.2 \\
0.15 & 0.40 & 0.3 \\
0.19 & 0.89 & 0.12 \\
0.26 & 0.63 & 0.06 \\
\hline
\end{tabular}

Table of parameters used in the theory, deduced by fitting the experimental data of the reflectance.

Supplementary Fig. 7 shows the comparison between the model and the experiment for the intensity noise at $\Delta=0.15 \mathrm{meV}$. The bistability curve of the reflectance and the corresponding intensity noise dependence on the pump power for a pump-polariton detuning of $0.26 \mathrm{meV}$ are shown in Supplementary Fig. 8 and in Supplementary Fig. 9, respectively.

\section{References}

1. Loo, V. et al. Optical nonlinearity for few-photon pulses on a quantum dot-pillar cavity device. Phys. Rev. Lett. 109, 166806 (2012).

2. Claudon, J. et al. A highly efficient single-photon source based on a quantum dot in a photonic nanowire. Nat. Photon. 4, 174-177 (2010).

3. Dousse, A. et al. Ultrabright source of entangled photon pairs. Nature 466, 217-220 (2010).

4. Kira, M., Jahnke, F. \& Koch, S. W. Quantum theory of secondary emission in optically excited semiconductor quantum wells. Phys. Rev. Lett. 82, 3544-3547 (1999).

5. Carmichael, H. J. \& Walls, D. F. Proposal for the measurement of the resonant Stark effect by photon correlation techniques. J. Phys. B 9, 43-48 (1976).

6. Kimble, H. J., Dagenais, M. \& Mandel, L. Photon antibunching in resonance fluorescence. Phys. Rev. Lett. 39, 691-695 (1977).

7. Slusher, R. E., Hollberg, L. W., Yurke, B., Mertz, J. C. \& Valley, J. F. Observation of squeezed states generated by four-wave mixing in an optical cavity. Phys. Rev. Lett. 55, 2409-2412 (1985).

8. Liew, T. \& Savona, V. Multipartite polariton entanglement in semiconductor microcavities. Phys. Rev. A 84, 032301 (2011).

9. Liew, T. \& Savona, V. Quantum entanglement in nanocavity arrays. Phys. Rev. A 85, 050301(R) (2012).

10. Fox, A. M., Baumberg, J. J., Dabbicco, M., Huttner, B. \& Ryan, J. F. Squeezed light generation in semiconductors. Phys. Rev. Lett. 74, 1728-1731 (1995).

11. Karr, J. Ph., Baas, A., Houdre, R. \& Giacobino, E. Squeezing in semiconductor microcavities in the strong coupling regime. Phys. Rev. A 69, 031802(R) (2004),

12. Baas, A., Karr, J. Ph., Eleuch, H. \& Giacobino, E. Optical bistability in semiconductor microcavities. Phys. Rev. A 69, 023809 (2004).

13. Saba, M. et al. High-temperature ultrafast polariton parametric amplification in semiconductor microcavities. Nature 414, 731-735 (2001).

14. Weisbuch, C., Nishioka, M., Ishikawa, A. \& Arakawa, Y. Observation of the coupled exciton-photon mode splitting in a semiconductor quantum microcavity. Phys. Rev. Lett. 69, 3314-3317 (1992)

15. Carusotto, I. \& Ciuti, C. Quantum fluids of light. Rev. Mod. Phys. 85, 299-366 (2013).

16. Kasprzak, J. et al. Bose-Einstein condensation of exciton polaritons. Nature 443, 409-414 (2006).

17. Amo, A. et al. Collective fluid dynamics of a polariton condensate in a semiconductor microcavity. Nature 457, 291-295 (2009).

18. Amo, A. et al. Superfluidity of polaritons in semiconductor microcavities. Nat. Phys. 5, 805-810 (2009).

19. Amo, A. et al. Polariton superfluids reveal quantum hydrodynamic solitons. Science 332, 1167-1170 (2011).

20. Sich, M. et al. Observation of bright polariton solitons in a semiconductor microcavity. Nat. Photon. 6, 50-55 (2011).

21. Hivet, R. et al. Half-solitons in a polariton quantum fluid behave like magnetic monopoles. Nat. Phys. 8, 724-728 (2012).

22. Lagoudakis, K. G. et al. Observation of half-quantum vortices in an excitonpolariton condensate. Science 326, 974-976 (2009).

23. Tosi, G. et al. Geometrically locked vortex lattices in semiconductor quantum fluids. Nat. Commun. 3, 1243 (2012).

24. Savvidis, P. G. et al. Angle-resonant stimulated polariton amplifier. Phys. Rev. Lett. 84, 1547-1550 (2000).

25. Stevenson, R. M. et al. Continuous wave observation of massive polariton redistribution by stimulated scattering in semiconductor microcavities. Phys. Rev. Lett. 85, 3680-3683 (2000).

26. Messin, G. et al. Parametric polariton amplification in semiconductor microcavities. Phys. Rev. Lett. 87, 127403 (2001).

27. Tassone, F. \& Yamamoto, Y. Lasing and squeezing of composite bosons in a semiconductor microcavity. Phys. Rev. A 62, 063809 (2000). 
28. Schwendimann, P., Ciuti, C. \& Quattropani, A. Statistics of polaritons in the nonlinear regime. Phys. Rev. B 68, 165324 (2003).

29. Bamba, M., Pigeon, S. \& Cuiti, C. Quantum squeezing generation versus photon localization in a disordered planar microcavity. Phys. Rev. Lett. 104, 213604 (2010)

30. Bloch, J. et al. Strong-coupling regime in pillar semiconductor microcavities. Superlattices Microstructures 22, 371-374 (1997).

31. Bajoni, D. et al. Polariton laser using single micropillar GaAs-GaAlAs semiconductor cavities. Phys. Rev. Lett. 100, 047401 (2008).

32. Hilico, L., Fabre, C., Reynaud, S. \& Giacobino, E. Linear input-output method for quantum fluctuations in optical bistability with two-level atoms. Phys. Rev. A 46, 4397-4406 (1992).

33. Romanelli, M., Karr, J. Ph., Leyder, C., Giacobino, E. \& Bramati, A. Two-mode squeezing in polariton four-wave mixing. Phys. Rev. B 82, 155313 (2010).

\section{Acknowledgements}

This work was partly supported by the FP7 ITN 'Clermont4' (235114), by the ANR projects PNANO-07-005 'GEMINI’ and Quandyde (ANR-11-BS10-001), by the French
RENATECH network and the KAKENHI 24-632 grant. The authors are grateful to J. Ph. Karr and Q. Glorieux for useful discussions.

\section{Author contributions}

All authors contributed to all aspects of this work.

\section{Additional information}

Supplementary Information accompanies this paper at http://www.nature.com/ naturecommunications

Competing financial interests: The authors declare no competing financial interests.

Reprints and permission information is available online at http://npg.nature.com/ reprintsandpermissions/

How to cite this article: Boulier, T. et al. Polariton-generated intensity squeezing in semiconductor micropillars. Nat. Commun. 5:3260 doi: 10.1038/ncomms4260 (2014) 